\title{
Protective effect of Pyropia yezoensis glycoprotein on chronic ethanol consumption-induced hepatotoxicity in rats
}

\author{
JEONG-WOOK CHOI ${ }^{1}$, IN-HYE KIM ${ }^{2}$, YOUNG-MIN KIM ${ }^{1}$, MIN-KYEONG LEE ${ }^{1}$, \\ YOUN-HEE $\mathrm{CHOI}^{2}$ and TAEK-JEONG NAM ${ }^{1,2}$ \\ ${ }^{1}$ Department of Food and Life Science, Pukyong National University, Busan 608-737; ${ }^{2}$ Institute of Fisheries Science, \\ Pukyong National University, Busan 619-911, Republic of Korea
}

Received September 15, 2015; Accepted September 20, 2016

DOI: $10.3892 / \mathrm{mmr} .2016 .5820$

\begin{abstract}
The present study investigated the protective effect of Pyropia yezoensis glycoprotein (PYGP) against chronic ethanol consumption-mediated hepatotoxicity in rats. Male Sprague-Dawley rats $(n=20 ; 6$ weeks old $)$ were randomly divided into four groups. The rats in each group were treated for 30 days with the following: i) $\mathrm{CON}$ group, distilled water only; ii) $\mathrm{EtOH}$ group, $20 \%$ ethanol $3.7 \mathrm{~g} / \mathrm{kg} / \mathrm{BW}$; iii) $\mathrm{EtOH}+150$ group, $20 \%$ ethanol $3.7 \mathrm{~g} / \mathrm{kg} / \mathrm{BW}+\mathrm{PYGP} 150 \mathrm{mg} / \mathrm{kg} / \mathrm{BW}$; iv) EtOH+300 group, $20 \%$ ethanol $3.7 \mathrm{~g} / \mathrm{kg} / \mathrm{BW}+\mathrm{PYGP} 300 \mathrm{mg} / \mathrm{kg} / \mathrm{BW}$. EtOH, PYGP and water were orally administered. The rats were sacrificed after 30 days, and blood and liver samples were collected for analysis. Treatment with ethanol caused significant elevation of serum levels of glutamic oxaloacetic transaminase (GOT) and glutamic pyruvic transaminase (GPT). Furthermore, inhibition of the antioxidant defense system in the liver, including glutathione (GSH), glutathione peroxidase (GSH-px) and catalase (CAT) was observed. However, co-administration with PYGP recovered the antioxidant defense system, and the serum levels of GOT and GPT. PYGP was shown to attenuate ethanol toxicity via the inactivation of mitogen-activated protein kinases (MAKPs). PYGP suppressed the overexpression of cytochrome P450 2E1 (CYP2E1), inducible nitric oxide synthase and cyclooxygenase-2. These results suggested that the protective effect of PYGP was associated with antioxidant activities, MAPKs and the CYP2E1 signaling pathway.
\end{abstract}

\section{Introduction}

Chronic ethanol consumption-induced alcoholic liver disease is one of the most common causes of liver cancer and

Correspondence to: Professor Taek-Jeong Nam, Department of Food and Life Science, Pukyong National University, 599-1, Daeyeon 3-dong, Nam-gu, Busan 608-737, Republic of Korea

E-mail:namtj@pknu.ac.kr

Key words: chronic ethanol consumption, Pyropia yezoensis, glycoprotein, liver injury cancer-associated mortality (1). Ethanol generates several harmful products, including reactive oxygen species (ROS), acetaldehyde (ADH) and cytochrome P450 2E1 (CYP2E1) during metabolism (2).

The CYP2E1 generated during ethanol metabolism generates reactive oxygen species and ADH (3). Ethanol consumption-induced liver pathology is correlated with the expression of CYP2E1 (4). The overexpression of CYP2E1 promotes lipid oxidation and oxidative stress in the liver (5).

ROS have been reported to affect protein oxidation, lipid oxidation, damage to DNA, enzyme inactivation and the depletion of various antioxidant enzymes (6-8). These results can lead early stages of the liver disease and dysfunction (9).

Glutathione (GSH), glutathione peroxidase (GSH-px) and catalase (CAT) are antioxidants, which affect the anti-oxidative system (10). The enzymatic antioxidant system includes superoxide dismutase (SOD), CAT and GSH-px (11). Nonenzymatic antioxidants consist of GSH, and vitamins A, $\mathrm{C}$ and $\mathrm{E}$ (12). The antioxidant system can assist in eliminating ROS and oxidative stress (13).

To date, several studies have shown that the mitogen-activated protein kinase (MAPK) family is crucial in cellular systems, including cell proliferation, cell differentiation, development, apoptosis and inflammatory responses (14-17). MAPKs consist of c-jun N-terminal kinase (JNK), p38 MAP kinase and extracellular signal-regulated kinase (ERK). Ethanol affects MAPKs in various cells and organ systems, which consequently show different pathologic consequences (14).

Chronic ethanol consumption upregulates the levels of inducible nitric oxide (NO) synthase (iNOS) and the protein expression levels of cyclooxygenase (COX)-2 in liver tissues (18). This protein leads to induction of the inflammatory response and oxidative stress (19). The increased expression of iNOS promotes the production of $\mathrm{NO}$ and the COX-2 protein, which subsequently leads to the release of pro-inflammatory cytokines (20).

Pyropia yezoensis, a species of marine algae, has long been consumed in Korea, Japan and China. It has a range of biological activities, including cell proliferation (21), antioxidation (22) and antiinflammatory effects (23). In the present study, the in vivo protective effect of $P$. yezoensis on chronic ethanol consumption-induced liver injury was investigated in mice. 


\section{Materials and methods}

Preparation of $P$. yezoensis glycoprotein (PYGP). Dried $P$. yezoensis was purchased in the Republic of Korea in 2014 (Suhyup, South Korea) and was homogenized using a blender. The $P$. yezoensis powder $(40 \mathrm{~g})$ was diluted 1:1 with distilled water and stirred for $4 \mathrm{~h}$ at room temperature. The solution was centrifuged $\left(3,000 \times \mathrm{g}\right.$ at $4^{\circ} \mathrm{C}$ for $\left.20 \mathrm{~min}\right)$ and vacuum filtered, following which triple volumes of ethanol (extract:ethanol, 1:3) were added. After $24 \mathrm{~h}$ at $4^{\circ} \mathrm{C}$, the solution was filtered and concentrated using rotary evaporation at $40^{\circ} \mathrm{C}$. The concentrated solution was divided into $1.5 \mathrm{ml}$ tubes, freeze-dried, and stored at $-70^{\circ} \mathrm{C}$ until use.

Experimental animals. Male Sprague-Dawley rats $(\mathrm{n}=20$; 6 weeks old) were purchased from Samtaco (Osan, South Korea). The rats were allowed to adapt to laboratory conditions for 1 week (temperature: $23 \pm 3^{\circ} \mathrm{C}, 12 \mathrm{~h}$ light $/ 12 \mathrm{~h}$ dark cycle, $50 \%$ humidity) with free access to water and food. Animal studies were conducted in accordance with the Animal Ethics Committee of Pukyong National University (Busan, South Korea).

Experimental design. The animals were randomly divided into four groups of five rats, as follows: Control rats, which received distilled water only (CON group); rats administered with $20 \%$ ethanol $(3.7 \mathrm{~g} / \mathrm{kg} / \mathrm{BW}$; EtOH group); rats administered with $20 \%$ ethanol $(3.7 \mathrm{~g} / \mathrm{kg} / \mathrm{BW})+\mathrm{PYGP}$ (150 mg/kg/BW; EtOH+150 group); and rats administered with $20 \%$ ethanol $(3.7 \mathrm{~g} / \mathrm{kg} / \mathrm{BW})+\mathrm{PYGP}(300 \mathrm{mg} / \mathrm{kg} / \mathrm{BW}$; $\mathrm{EtOH}+300$ group). The PYGP and ethanol were administered orally once per day for 30 days. The animals in all groups were sacrificed for blood and liver collection at the end of experimental period (day 31). Blood was collected immediately, and the livers were frozen in liquid nitrogen and stored at $-70^{\circ} \mathrm{C}$ until use.

Biochemical indicators of liver function. Blood samples were centrifuged at $3,000 \mathrm{x} g$ for $20 \mathrm{~min}$ at $4^{\circ} \mathrm{C}$ to collect serum, which was stored at $-20^{\circ} \mathrm{C}$ until further analysis. Chronic hepatic damage was measured by detecting the serum levels of GOT and GPT using an enzymatic analysis kit (Asan Pharmaceuticals Co., Ltd., Hwasung, South Korea) according to the manufacturer's protocol. The absorbance was measured using a UV spectrometer (Ultrospec 2100 pro; GE Healthcare Life Sciences, Cambridge, UK).

Antioxidant enzyme measurement. The activities of antioxidant enzymes, including CAT, GSH and GSH-px, in the homogenized liver samples were measured using a CAT assay kit, GSH assay kit and GSH-px assay kit, respectively, according to the manufacturer's protocols (Cayman Chemical Company, Ann Arbor, MI, USA). The absorbance was measured using a microplate reader (Benchmark Plus 10730; Bio-Rad Laboratories, Inc., Hercules, CA, USA).

Western blot analysis. The liver tissue protein was homogenized in lysis buffer containing $150 \mathrm{mM}$ sodium chloride, $50 \mathrm{mM}$ Tris- $\mathrm{HCl}$ ( $\mathrm{pH} 7.5), 0.5 \%$ sodium deoxycholate, $0.1 \%$ sodium dodecyl sulfate, $1 \%$ triton $\mathrm{X}-100$ and $2 \mathrm{mM}$ ethylenediaminetetra-acetic acid (Intron Biotechnology, Inc., Seoul, South Korea) with inhibitors (1 $\mathrm{mM} \mathrm{Na}_{3} \mathrm{VO}_{4}$, $1 \mu \mathrm{g} / \mathrm{ml}$ aprotinin, $1 \mu \mathrm{g} / \mathrm{ml}$ leupeptin, $1 \mu \mathrm{g} / \mathrm{ml}$ pepstatin A and $1 \mathrm{mM}$ PMSF; (Sigma-Aldrich; Merck Millipore, Darmstadt, Germany). The protein levels were determined using a Bichinchominic Acid Assay kit (Pierce; Thermo Fisher Scientific, Inc., Waltham, MA, USA). Equal amounts of protein $(20 \mu \mathrm{g})$ were separated via $10-15 \%$ SDS-PAGE and then transferred onto a polyvinylidene fluoride membrane (EMD Millipore, Billerica, MA, USA). The transferred membrane was blocked with $1 \%$ bovine serum albumin (BSA) in TBS-T containing $10 \mathrm{mM}$ Tris- $\mathrm{HCl}$ ( $\mathrm{pH} \mathrm{7.5),}$ $150 \mathrm{mM} \mathrm{NaCl}$ and $0.1 \%$ Tween 20 (USB, Cleveland, $\mathrm{OH}$, USA). Following blocking, the membranes were incubated for $4 \mathrm{~h}$ at room temperature with the following primary antibodies: Rabbit anti-rat ERK IgG polyclonal antibody (diluted 1:1,000 with BSA/TBS-T; cat. no. sc-94), rabbit anti-rat phosphorylated (p)-ERK IgG polyclonal antibody (diluted 1:1,000 with BSA/TBS-T; cat. no. sc-7383), mouse anti-rat JNK IgG monoclonal antibody (diluted 1:1,000 with BSA/TBS-T; cat. no. sc-7345), mouse anti-rat p-JNK IgG monoclonal antibody (diluted 1:1,000 with BSA/TBS-T; cat. no. sc-6254), rabbit anti-rat p38 IgG polyclonal antibody (diluted 1:1,000 with BSA/TBS-T; cat. no. sc-7149), mouse anti-rat p-p38 IgG monoclonal antibody (diluted 1:1,000 with BSA/TBS-T; cat. no. sc-7973), mouse anti-rat iNOS IgG polyclonal antibody (diluted 1:1,000 with BSA/TBS-T; cat. no. sc-650), goat anti-rat COX-2 IgG polyclonal antibody (diluted 1:1,000 with BSA/TBS-T; cat. no. sc-1745), rabbit anti-rat CYP2E1 IgG polyclonal antibody (diluted 1:1,000 with BSA/TBS-T; cat. no. sc-133491) and rabbit anti-rat GAPDH IgG polyclonal antibody (diluted 1:1,000 with BSA/TBS-T; cat. no. sc-25778), all from Santa Cruz Biotechnology, Inc. (Dallas, TX, USA). The membranes were then incubated with peroxidase-conjugated goat (cat. no. A50-101P), mouse (cat. no. A90-116P) and rabbit (cat. no. A120-101P) secondary antibodies (1:10,000; GE Healthcare Life Sciences, Little Chalfont, UK) for $1 \mathrm{~h}$ at room temperature. Antibody binding was visualized using Super Signal West Pico Stable Peroxide solution and Super Signal West Pico Luminol/Enhancer solution (Thermo Fisher Scientific, Inc.). The signal was monitored using X-ray film (Kodak, Rochester, NY, USA), and a developer and fixer twin pack (Kodak).

Statistical analysis. Values are presented as the mean + standard deviation and data were analyzed using SPSS version 10.0 software (SPSS, Inc., Chicago, IL, USA) using one-way analysis of variance followed by a Duncan's multiple range test. $\mathrm{P}<0.05$ was considered to indicate a statistically significant difference.

\section{Results}

Hepatoprotective effect of PYGP against chronic ethanol consumption. In hepatotoxicity, serum levels of GOT and GPT are increased by liver injury or liver cell destruction (24). The results of the present study revealed that the levels of GOT and GPT were significantly increased in the ethanol group, compared with those of the control group. However, the groups co-administered with PYGP showed decreased levels of GOT and GPT (Fig. 1A and B). 
A

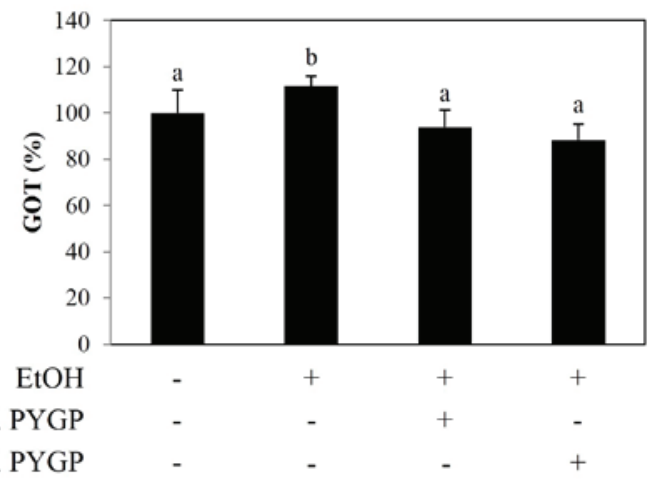

B

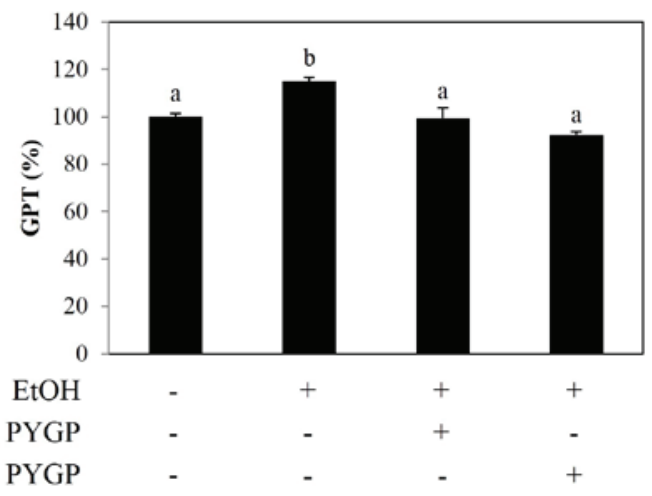

Figure 1. Levels of (A) GOT and (B) GPT in the serum of control and experimental rat groups. Values are presented as the mean + standard deviation. Groups with different letters $(\mathrm{a}$ and $\mathrm{b})$ are significantly different $(\mathrm{P}<0.05)$. EtOH, ethanol; GOT, glutamic oxaloacetic transaminase; GPT, glutamic pyruvic transaminase; CON, control; 150, $150 \mathrm{mg} / \mathrm{kg}$ Pyropia yezoensis glycoprotein, $300,300 \mathrm{mg} / \mathrm{kg}$ Pyropia yezoensis glycoprotein.

Antioxidant enzyme activity in the rat liver. The activities of the CAT, GSH and GSH-px antioxidant enzymes were markedly decreased in the ethanol-only group, compared with the control group. By contrast, the activities of GSH and GSH-px were restored in the group co-administered with ethanol and PYGP (300 mg/kg; Fig. 2A and B). In addition, the activity of CAT was significantly increased by co-administration with PYGP in a concentration-dependent manner (Fig. 2C).

Ethanol-induced phosphorylation of MAPK is inhibited by $P Y G P$. To examine whether PYGP can inhibit the phosphorylation of MAPK, the present study used western blot assays. The results revealed that ethanol induced the phosphorylation of ERK, JNK and p38, compared with the control group. PYGP was effective at inhibiting the ethanol-induced protein phosphorylation of ERK, JNK and p38. By contrast, ethanol and PYGP had no effect on the total protein expression of ERK, JNK or p38. These result suggested that PYGP inhibited the ethanol-induced phosphorylation of MAPK (Fig. 3).

Effects of PYGP on the expression of COX-2, iNOS and $C Y P 2 E 1$. Chronic ethanol consumption is known to increase the protein expression levels of iNOS, COX-2 and CYP2E1. These proteins are associated with liver inflammation and cell injury $(19,20)$. In the present study, chronic ethanol consumption upregulated the protein expression levels of iNOS, COX-2 and CYP2E1. By contrast, when the rats were co-administered with ethanol and PYGP, the protein expression levels of iNOS, COX-2 and CYP2E1 were markedly downregulated. These results confirmed that PYGP was important in the suppression of chronic ethanol-induced protein expression of iNOS, COX-2 and CYP2E1 (Fig. 4).

\section{Discussion}

Ethanol consumption-induced pathogenesis is complicated. It is associated with oxidative stress, ROS generation and alterations in the innate immune response via ethanol metabolism (25-27). Chronic ethanol consumption in humans leads to serious liver problems, including fibrosis, cirrhosis and hepatocellular carcinoma (28). The increased levels of GOT and GPT as a result of liver injury are commonly used as a measure of hepatotoxicity. In the present study, chronic ethanol consumption increased the serum levels of GOT and GPT, whereas co-administration with PYGP attenuated this increase, resulting in levels similar to those measured in the control group.

Chronic ethanol consumption induces the loss of antioxidant or the diminution of enzyme activities, including those of GSH, GSH-px and CAT (29). GSH is a tripeptide and effectively scavenges ROS and free radicals (30). GSH-px acts as a catalyst in the reduction of $\mathrm{H}_{2} \mathrm{O}_{2}$ and diverse hydroperoxides, with $\mathrm{GSH}$ acting as an electron donor (31). CAT is important in the decomposition of $\mathrm{H}_{2} \mathrm{O}_{2}$ and the formation of $\mathrm{H}_{2} \mathrm{O}$ and $\mathrm{O}_{2}$ (32). In the present study, the activities of the antioxidant enzymes, GSH, GSH-px and CAT, were significantly decreased by chronic ethanol consumption, whereas co-administration with PYGP increased the activities of these enzymes, compared with the ethanol-only treatment group.

MAPKs are serine-threonine kinases, which are essential in intracellular signaling, including cell proliferation, differentiation, transformation, survival and death (33). Ethanol consumption activates the MAPK cascade via protein phosphorylation (34). In particular, these proteins regulate oxidative stress in ethanol-induced hepatotoxicity (35). In the present study, the results showed that ethanol consumption caused the phosphorylation of ERK, JNK and p38 in the rat liver, whereas co-administration with PYGP attenuated the levels of phosphorylation.

Chronic ethanol consumption increases the protein expression levels of COX-2 and iNOS in the liver (36). These proteins are associated with the ethanol-induced liver inflammatory response (37). NO is a reactive oxidizing agent, and the synthesis of NO is associated with the expression of iNOS (38). Although predominantly involved in the protective effect against bacteria, parasites and tumor cells, the overexpression of NO causes damage to organs (39). COX-2 is associated with several biological response, including inflammation, carcinogenesis and hepatic fibrogenesis (40). In alcoholic liver disease, the expression of COX-2 is increased in Kupffer cells (41). The increased expression of COX-2 promotes lipid peroxidation, endotoxins, synthesis of tumor necrosis factor- $\alpha$ and thromboxane $\mathrm{B}_{2}$ $\left(\mathrm{TXB}_{2}\right) . \mathrm{TXB}_{2}$, in particular, is associated with serious alcoholic liver disease (42). In the present study, the protein expression levels of iNOS and COX-2 were increased by chronic ethanol 
A

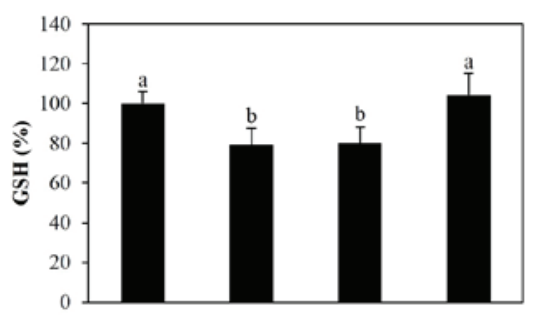

$\mathrm{EtOH}$

$150 \mathrm{mg} / \mathrm{kg}$ B.W. PYGP $300 \mathrm{mg} / \mathrm{kg}$ B.W. PYGP
B

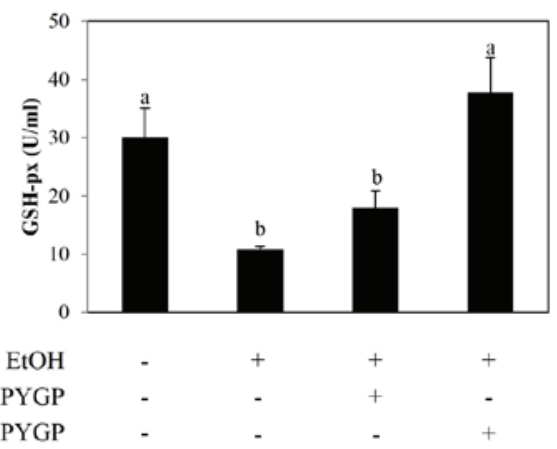

C

$150 \mathrm{mg} / \mathrm{kg}$ B.W. PYGP $300 \mathrm{mg} / \mathrm{kg}$ B.W. PYGP

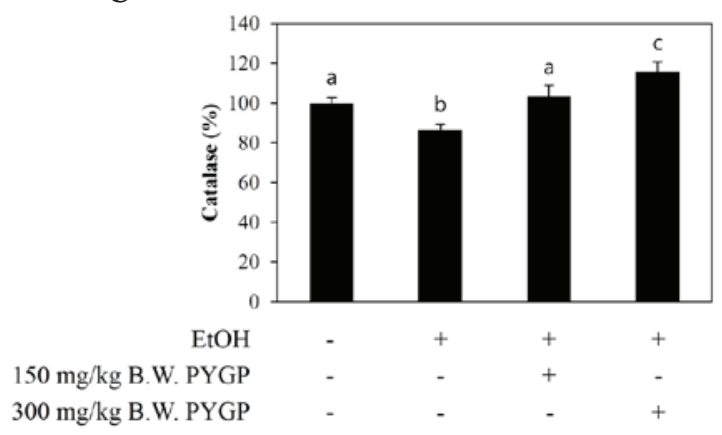

Figure 2. Levels of the antioxidant enzymes, GSH, GSH-px and CAT, in the livers of rats in control and experimental groups. (A) GSH, (B) GSH-px and (C) CAT. Values are presented as the mean + standard deviation. Groups with different letters $(\mathrm{a}, \mathrm{b}$ and $\mathrm{c})$ are significantly different from each other $(\mathrm{P}<0.05)$. EtOH, ethanol; GSH, glutathione; GSH-px, glutathione peroxidase; CAT, catalase; CON, control; 150, $150 \mathrm{mg} / \mathrm{kg}$ Pyropia yezoensis glycoprotein, 300, $300 \mathrm{mg} / \mathrm{kg}$ Pyropia yezoensis glycoprotein.

p-p38
p38
p-JNK
JNK
p-ERK
ERK
GAPDH

PYGP $(\mathrm{mg} / \mathrm{kg})$ $\mathrm{EtOH}$

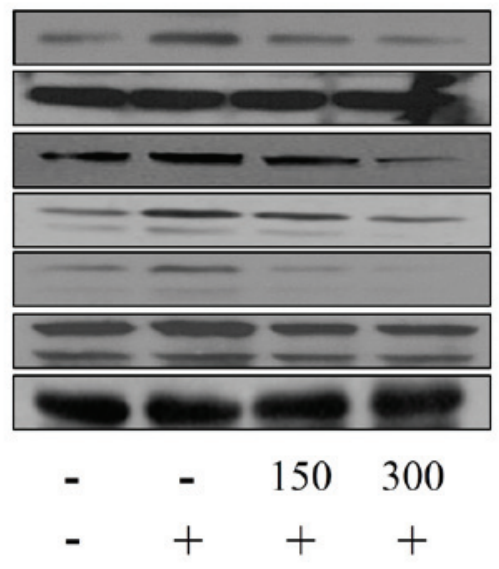

Figure 3. Expression of proteins of the mitogen-activated protein kinase family in the livers of rats in the control and experimental groups. Livers were collected and lysed, and western blot analysis was performed to determine the total protein expression and phosphorylation levels of ERK, JNK and p38. PYGP, Pyropia yezoensis glycoprotein; EtOH, ethanol; ERK, extracellular signal-regulated kinase; JNK, c-jun N-terminal kinase; p-, phosphorylated.

consumption, whereas co-administration with PYGP attenuated the levels of expression, compared with the ethanol only group.

Chronic ethanol consumption promotes the production of CYP2E1 and generates increased levels of ROS, including $\mathrm{H}_{2} \mathrm{O}_{2}$ and $\mathrm{O}_{2}(43,44)$. CYP2E1 catalyzes the oxidation of

\section{iNOS \\ COX-2 \\ CYP2E1 \\ GAPDH}

PYGP $(\mathrm{mg} / \mathrm{kg})$

$\mathrm{EtOH}$

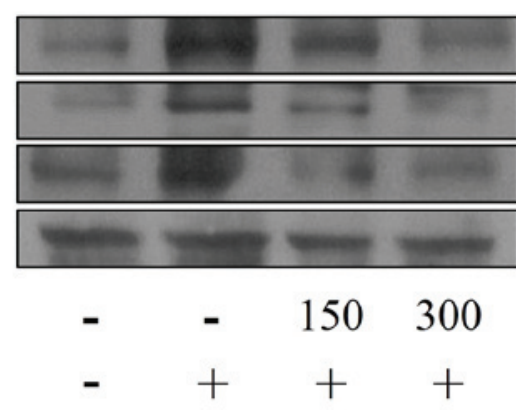

Figure 4. Protein expression of iNOS, COX-2 and CYP2E1 in the livers of rats in the control and experimental groups. Livers were collected and lysed, and western blot analysis was performed to determine the total protein expression of iNOS, COX-2 and CYP2E1. PYGP, Pyropia yezoensis glycoprotein; EtOH, ethanol; iNOS, inducible nitric oxide synthase; COX-2, cyclooxygenase-2; CYP2E1, cytochrome P450 2E1.

small quantities of ethanol ( 10\%) into ADH (45), and ADH is considered to be a major toxin in ethanol-induced liver injury, inflammation and extracellular matrix (46). In the present study, the rats exposed to chronic ethanol consumption showed higher levels of CYP2E1 enzyme production in the liver, compared with the rats in the control group. The co-administration of ethanol with PYGP showed inhibition in the production of CYP2E1.

In conclusion, the present study demonstrated that chronic ethanol consumption induced hepatotoxicity and inhibited the levels of antioxidants, including GSH, 
GSH-px and CAT, in the liver. In addition, chronic ethanol consumption promoted the overexpression of iNOS, COX-2 and CYP2E1, and the overactivation of ERK, JNK and p38. PYGP prevented chronic ethanol consumption-induced hepatotoxicity in the rats via the downregulation of MAPKs, iNOS, COX-2 and CYP2E1. These results suggested that PYGP may offer potential for use as a novel treatment against chronic ethanol hepatotoxicity.

\section{Acknowledgements}

This study was supported by the Fishery Commercialization Technology Development Program through the Korea Institute of Planning and Evaluation of Technology in Food, Agriculture, Forestry and Fisheries (iPET) funded by the Ministry of Oceans and Fisheries (grant no. 2012300734).

\section{References}

1. Jemal A, Bray F, Center MM, Ferlay J, Ward E and Forman D: Global cancer statistics. CA Cancer J Clin 61: 69-90, 2011.

2. Saravanan R, Viswanathan P and Pugalendi KV: Protective effect of ursolic acid on ethanol-mediated experimental liver damage in rats. Life Sci 78: 713-718, 2006.

3. Cederbaum AI: Role of CYP2E1 in ethanol-induced oxidant stress, fatty liver and hepatotoxicity. Dig Dis 28: 802-811, 2010.

4. Morgan K, French SW and Morgan TR: Production of a cytochrome P450 2E1 transgenic mouse and initial evaluation of alcoholic liver damage. Hepatology 36: 122-134, 2002.

5. Castillo T, Koop DR, Kamimura S, Triadafilopoulos G and Tsukamoto H: Role of cytochrome P-450 2E1 in ethanol-, carbon tetrachloride- and iron-dependent microsomal lipid peroxidation. Hepatology 16: 992-996, 1992.

6. Rouach H, Fatacciolo V, Gentil M, French SW, Morimoto M and Nordman R: Effect of chronic ethanol feeding on lipid peroxidation and protein oxidation in relation to liver pathology. Hepatology 25: 351-355, 1997.

7. Fernandez-Checa JC, Ookhtens M and Kaplowitz N: Effect of chronic ethanol feeding on rat hepatocytic glutathione: Compartmentation, efflux, and response to incubation with ethanol. J Clin Invest 80: 57-62, 1987.

8. Nordmann R, Ribière $\mathrm{C}$ and Rouach $\mathrm{H}$ : Implication of free radical mechanisms in ethanol-induced cellular injury. Free Radic Biol Med 12: 219-240, 1992.

9. Liu LL, Gong LK, Qi XM, Cai Y, Wang H, Wu XF, Xiao Y and Ren J: Altered expression of cytochrome P450 and possible correlation with preneoplastic changes in early stage of rat hepatocarcinogenesis. Acta Pharmacol Sin 26: 737-744, 2005.

10. Matés JM, Pérez-Gómez C and Núñez de Castro I: Antioxidant enzymes and human diseases. Clin Biochem 32: 595-603, 1999.

11. Jurczuk M, Brzóska MM, Moniuszko-Jakoniuk J, Gałazyn-Sidorczuk $M$ and Kulikowska-Karpińska E: Antioxidant enzymes activity and lipid peroxidation in liver and kidney of rats exposed to cadmium and ethanol. Food Chem Toxicol 42: 429-438, 2004.

12. Martin KR and Barrett JC: Reactive oxygen species as double-edged swords in cellular processes: Low-dose cell signaling versus high-dose toxicity. Hum Exp Toxicol 21: $71-75,2002$

13. Scott RB, Reddy KS, Husain K, Schlorff EC, Rybak LP and Somani SM: Dose response of ethanol on antioxidant defense system of liver, lung, and kidney in rat. Pathophysiology 7: 25-32, 2000.

14. Das SK and Vasudevan DM: Alcohol-induced oxidative stress. Life Sci 81: 177-187, 2007.

15. Venugopal SK, Chen J, Zhang Y, Clemens D, Follenzi A and Zern MA: Role of MAPK phosphatase-1 in sustained activation of JNK during ethanol-induced apoptosis in hepatocyte-like VL-17A cells. J Biol Chem 282: 31900-31908, 2007.

16. Cross TG, Scheel-Toellner D, Henriquez NV, Deacon E, Salmon M and Lord JM: Serine/threonine protein kinases and apoptosis. Exp Cell Res 256: 34-41, 2000.
17. Pearson G, Robinson F, Beers Gibson T, Xu BE, Karandikar M, Berman K and Cobb MH: Mitogen-activated protein (MAP) kinase pathways: Regulation and physiological functions. Endocr Rev 22: 153-183, 2001.

18. Nanji AA, Jokelainen K, Tipoe GL, Rahemtulla A, Thomas P and Dannenberg AJ: Curcumin prevents alcohol-induced liver disease in rats by inhibiting the expression of NF-kappa B-dependent genes. Am J Physiol Gastrointest Liver Physiol 284: G321-G327, 2003.

19. Hseu YC, Wu FY, Wu JJ, Chen JY, Chang WH, Lu FJ, Lai YC and Yang HL: Anti-inflammatory potential of Antrodia camphorata through inhibition of iNOS, COX-2 and cytokines via the NF-kappa B pathway. Int Immunopharmacol 5: 1914-1925, 2005.

20. Surh YJ, Chun KS, Cha HH, Han SS, Keum YS, Park KK and Lee SS: Molecular mechanisms underlying chemopreventive activities of anti-inflammatory phytochemicals: Down-regulation of COX-2 and iNOS through suppression of NF-kappa B activation. Mutat Res 480: 243-268, 2001.

21. Lee MK, Kim IH, Choi YH, Choi JW, Kim YM and Nam TJ: The proliferative effects of Pyropia yezoensis peptide on IEC- 6 cells are mediated through the epidermal growth factor receptor signaling pathway. Int J Mol Med 35: 909-914, 2015.

22. Nakayama R, Tamura Y, Kikuzaki H and Nakatani N: Antioxidant effect of the constituents of Susabinori (Porphyra yezoensis). J Am Oil Chem Soc 76: 649-653, 1999.

23. Shin ES, Hwang HJ, Kim IH and Nam TJ: A glycoprotein from Porphyra yezoensis produces anti-inflammatory effects in liposaccharide-stimulated macrophages via the TLR4 signaling pathway. Int J Mol Med 28: 809-815, 2011.

24. Yamaguchi M, Tsurusaki Y, Misawa H, Inagaki S, Ma ZJ and Takahashi $\mathrm{H}$ : Potential role of regucalcin as a specific biochemical marker of chronic liver injury with carbon tetrachloride administration in rats. Mol Cell Biochem 241: 61-67, 2002.

25. Bailey SM and Cunningham CC: Contribution of mitochondria to oxidative stress associated with alcoholic liver disease. Free Radic Biol Med 32: 11-16, 2002.

26. Hines IN and Wheeler MD: Recent advances in alcoholic liver disease III. Role of the innate immune response in alcoholic hepatitis. Am J Physiol Gastrointest Liver Physiol 287: G310-G314, 2004.

27. Kumar KJ, Chu FH, Hsieh HW, Liao JW, Li WH, Lin JC, Shaw JF and Wang SY: Antroquinonol from ethanolic extract of mycelium of Antrodia cinnamomea protects hepatic cells from ethanol-induced oxidative stress through Nrf-2 activation. J Ethnopharmacol 136: 168-177, 2011.

28. Leung MT, Lu Y, Yan W, Morón-Concepción JA, Ward SC, Ge X, Conde de la Rosa L and Nieto N: Argininosuccinate synthase conditions the response to acute and chronic ethanol-induced liver injury in mice. Hepatology 55: 1596-1609, 2012.

29. Ostrowska J, Łuczaj W, Kasacka I, Różański A and Skrzydlewska E: Green tea protects against ethanol-induced lipid peroxidation in rat organs. Alcohol 32: 25-32, 2004.

30. Wu G, Fang YZ, Yang S, Lupton JR and Turner ND: Glutathione metabolism and its implications for health. J Nutr 134: 489-492, 2004.

31. Chang TS, Cho CS, Park S, Yu S, Kang SW and Rhee SG: Peroxiredoxin III, a mitochondrion-specific peroxidase, regulates apoptotic signaling by mitochondria. J Biol Chem 279: 41975-41984, 2004.

32. Vimal V and Devaki T: Hepatoprotective effect of allicin on tissue defense system in galactosamine/endotoxin challenged rats. J Ethnopharmacol 90: 151-154, 2004.

33. Wada T and Penninger JM: Mitogen-activated protein kinases in apoptosis regulation. Oncogene 23: 2838-2849, 2004.

34. Park HM, Kim SJ, Mun AR, Go HK, Kim GB, Kim SZ, Jang SI, Lee SJ, Kim JS and Kang HS: Korean red ginseng and its primary ginsenosides inhibit ethanol-induced oxidative injury by suppression of the MAPK pathway in TIB-73 cells. J Ethnopharmacol 141: 1071-1076, 2012.

35. Aroor AR and Shukla SD: MAP kinase signaling in diverse effects of ethanol. Life Sci 74: 2339-2364, 2004.

36. Tahir M, Rehman MU, Lateef A, Khan R, Khan AQ, Qamar W, Ali F, O'Hamiza O and Sultana S: Diosmin protects against ethanol-induced hepatic injury via alleviation of inflammation and regulation of TNF- $\alpha$ and NF- $\kappa \mathrm{B}$ activation. Alcohol 47: $131-139,2013$. 
37. Murakami A and Ohigashi H: Targeting NOX, INOS and COX-2 in inflammatory cells: Chemoprevention using food phytochemicals. Int J Cancer 121: 2357-2363, 2007.

38. Gardner CR, Heck DE, Yang CS, Thomas PE, Zhang XJ, DeGeorge GL, Laskin JD and Laskin DL: Role of nitric oxide in acetaminophen-induced hepatotoxicity in the rat. Hepatology 27: 748-754, 1998.

39. Quan J, Yin X and Xu H: Boschniakia rossica prevents the carbon tetrachloride-induced hepatotoxicity in rat. Exp Toxicol Pathol 63: 53-59, 2011.

40. Hu KQ: Cyclooxygenase 2 (COX2)-prostanoid pathway and liver diseases. Prostaglandins Leukot Essent Fatty Acids 69: 329-337, 2003.

41. Nanji AA, Miao L, Thomas P, Rahemtulla A, Khwaja S, Zhao S, Peters D, Tahan SR and Dannenberg AJ: Enhanced cyclooxygenase-2 gene expression in alcoholic liver disease in the rat. Gastroenterology 112: 943-951, 1997.
42. Nanji AA, Khettry U, Sadrzadeh SM and Yamanaka T: Severity of liver injury in experimental alcoholic liver disease. Correlation with plasma endotoxin, prostaglandin E2, leukotriene B4, and thromboxane B2. Am J Pathol 142: 367-373, 1993.

43. Zima T and Kalousová M: Oxidative stress and signal transduction pathways in alcoholic liver disease. Alcohol Clin Exp Res 29 (Suppl 11): S110-S115, 2005.

44. Lu Y and Cederbaum AI: CYP2E1 and oxidative liver injury by alcohol. Free Radic Biol Med 44: 723-738, 2008.

45. Ceni E, Mello T and Galli A: Pathogenesis of alcoholic liver disease: Role of oxidative metabolism. World J Gastroenterol 20: 17756-17772, 2014.

46. Seth D, Haber PS, Syn WK, Diehl AM and Day CP: Pathogenesis of alcohol-induced liver disease: Classical concepts and recent advances. J Gastroenterol Hepatol 26: 1089-1105, 2011. 